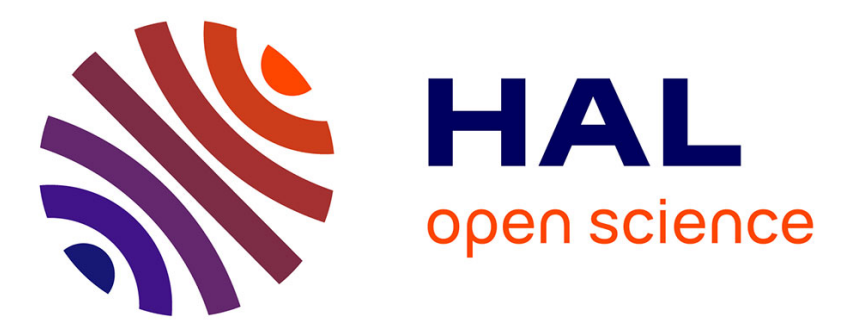

\title{
Influence of Al doping on the critical fields and gap values in magnesium diboride single crystals
}

Thierry Klein, L. Lyard, J. Marcus, C. Marcenat, P. Szabo, Z. Holanova, P. Samuely, W. Kang, H.-J. Kim, H.-S. Lee, et al.

\section{- To cite this version:}

Thierry Klein, L. Lyard, J. Marcus, C. Marcenat, P. Szabo, et al.. Influence of Al doping on the critical fields and gap values in magnesium diboride single crystals. Physical Review B: Condensed Matter and Materials Physics (1998-2015), 2006, 73, pp.224528. 10.1103/PhysRevB.73.224528 . hal-00957685

\section{HAL Id: hal-00957685 https://hal.science/hal-00957685}

Submitted on 11 Mar 2014

HAL is a multi-disciplinary open access archive for the deposit and dissemination of scientific research documents, whether they are published or not. The documents may come from teaching and research institutions in France or abroad, or from public or private research centers.
L'archive ouverte pluridisciplinaire $\mathbf{H A L}$, est destinée au dépôt et à la diffusion de documents scientifiques de niveau recherche, publiés ou non, émanant des établissements d'enseignement et de recherche français ou étrangers, des laboratoires publics ou privés. 


\title{
Influence of Al doping on the critical fields and gap values in magnesium diboride single crystals
}

\author{
T. Klein, ${ }^{1,2}$ L. Lyard, ${ }^{1}$ J. Marcus, ${ }^{1}$ C. Marcenat, ${ }^{3}$ P. Szabó,${ }^{4}$ Z. Hol'anová, ${ }^{4}$ P. Samuely, ${ }^{4}$ B. W. Kang, ${ }^{5}$ H-J. Kim, ${ }^{5}$ \\ H-S. Lee, ${ }^{5}$ H-K. Lee, ${ }^{5}$ and S-I. Lee ${ }^{5}$ \\ ${ }^{1}$ Laboratoire d'Etudes des Propriétés Electroniques des Solides, Centre National de la Recherche Scientifique, B.P. 166, \\ F-38042 Grenoble Cedex 9, France \\ ${ }^{2}$ Institut Universitaire de France and Université Joseph Fourier, B.P. 53, 38041 Grenoble Cedex 9, France \\ ${ }^{3}$ CEA-Grenoble, Département de Recherche Fondamental sur la Matière Condensée, F-38054 Grenoble Cedex 9, France \\ ${ }^{4}$ Centre of Low Temperature Physics IEP SAS \& FS UPJŠ, Watsonova 47, 04353 Košice, Slovakia \\ ${ }^{5}$ NVCRICS and Department of Physics, Pohang University of Science and Technology, Pohang 790-784, Republic of Korea
}

(Received 16 December 2005; revised manuscript received 17 May 2006; published 30 June 2006)

\begin{abstract}
The lower $\left(H_{c 1}\right)$ and upper $\left(H_{c 2}\right)$ critical fields of $\mathrm{Mg}_{1-x} \mathrm{Al}_{x} \mathrm{~B}_{2}$ single crystals (for $x=0,0.1$, and $\left.\gtrsim 0.2\right)$ have been deduced from local magnetization and specific heat measurements, respectively. We show that $H_{c 1}$ and $H_{c 2}$ are both decreasing with increasing doping content. The corresponding anisotropy parameter $\Gamma_{H_{c 2}}(0)$ $=H_{c 2}^{a b}(0) / H_{c 2}^{c}(0)$ value also decreases from $\sim 5$ in pure $\mathrm{MgB}_{2}$ samples down to $\sim 1.5$ for $x \geqslant 0.2$ whereas $\Gamma_{H_{c 1}}(0)=H_{c 1}^{c}(0) / H_{c 1}^{a b}(0)$ remains on the order of 1 in all samples. The small and large gap values have been obtained by fitting the temperature dependence of the zero-field electronic contribution to the specific heat to the two-gap model for the three $\mathrm{Al}$ concentrations. Very similar values have also been obtained by point contact spectroscopy measurements. The evolution of those gaps with Al concentration suggests that both band filling and interband scattering effects are present.
\end{abstract}

DOI: 10.1103/PhysRevB.73.224528

PACS number(s): 74.25.Dw, 74.25.Bt, 74.25.Ha, 74.50.+r

\section{INTRODUCTION}

It is now well established that $\mathrm{MgB}_{2}$ belongs to an original class of superconductors in which two weakly coupled bands with very different characters coexist (an almost isotropic $\pi$ band and a quasi-two-dimensional $\sigma$ band). This unique behavior has been rapidly confirmed by spectroscopy ${ }^{1}$ and specific heat ${ }^{2}$ measurements which both revealed the existence of two distinct superconducting gaps. One of the main consequence of this two-band superconductivity is a strong temperature dependence of the anisotropy of the upper critical field $\Gamma_{H_{c 2}}=H_{c 2}^{a b} / H_{c 2}^{c}$ (Refs. 3-6) ( $H_{c 2}^{a b}$ and $H_{c 2}^{c}$ being the upper critical fields parallel to the $a b$ planes and $c$ axis, respectively). On the other hand, the lower critical field $\left(H_{c 1}\right)$ is almost isotropic at low temperatures and the corresponding anisotropy $\Gamma_{H_{c 1}}=H_{c 1}^{c} / H_{c 1}^{a b}$ slightly increases with temperature merging with $\Gamma_{H_{c 2}}$ for $T \rightarrow T_{c}{ }^{7}$

The influence of chemical doping and/or impurity scattering on the physical properties has then been widely addressed in both carbon- $\left[\mathrm{Mg}\left(\mathrm{B}_{1-x} \mathrm{C}_{x}\right)_{2}\right]$ and aluminum$\left(\mathrm{Mg}_{1-x} \mathrm{Al}_{x} \mathrm{~B}_{2}\right)$ doped samples. Whereas all studies agree on a significant increase of the upper critical field in both directions due to strong impurity scattering in C-doped samples, ${ }^{8-10}$ no consensus was met in the latter system. On the one hand, transport measurements in single crystals of Al-doped samples recently suggested a decrease of both $H_{c 2}^{c}(T)$ and $H_{c 2}^{a b}(T)$ which has been analyzed within the dirtylimit two-gap theory. ${ }^{11}$ On the other hand, similar measurements in polycrystals rather suggested that the system remains in the clean limit ${ }^{12-14}$ with $H_{c 2}^{c}$ being roughly independent of $x$ and $H_{c 2}^{a b}$ decreasing for increasing doping content. ${ }^{14}$

Also, the evolution of the superconducting gaps with $\mathrm{Al}$ doping remains controversial. Whereas Gonnelli et al. ${ }^{15}$ suggested that the small gap rapidly decreases in single crystals for $x>0.09$ due to some phase segregation, Putti et al. ${ }^{13}$ suggested a progressive decrease of both gaps in polycrystals up to $x=0.3$. However, in this latter work both gaps have a $2 \Delta / k T_{c}$ ratio smaller than the BCS canonical 3.5 value which casts some doubt on the way the authors extracted the gap values from the experimental spectra.

In this paper, we report on specific heat, Hall probe magnetization, and point contact spectroscopy (PCS) measurements performed on $\mathrm{Mg}_{1-x} \mathrm{Al}_{x} \mathrm{~B}_{2}$ single crystals grown at the Pohang University of Science and Technology $(x=0,0.1$, and $\gtrsim 0.2$ ). The paper is divided as follows. As some uncertainty is related to the determination of $H_{c 2}$ from either magnetic or transport measurements, we performed specific heat $\left(C_{p}\right)$ measurements for both $H \| c$ and $H \| a b$. Indeed, $C_{p}$ probes the thermodynamic properties of the bulk of the sample and thus provides an unambiguous criterion for the determination of $H_{c 2}$; those measurements are presented in Sec. II. We show that $H_{c 2}^{c}(0)$ and $H_{c 2}^{a b}(0)$ both decrease with increasing $x$ and that the corresponding anisotropy $\Gamma_{H_{c 2}}(0)$ also decreases (being equal to $\sim 5, \sim 3$, and $\sim 1.5$ for $x=0,0.1$, and $\gtrsim 0.2$, respectively). As the $\pi$ band is rapidly filled up by a magnetic field, the $H_{c 2}(0)$ values are expected to be mainly determined by the parameters of the $\sigma$ band and, as discussed in Refs. 12 and 14, the decrease of $H_{c 2}^{a b}$ can then be attributed to the decrease of $\Delta_{\sigma}$ due to electronic doping and/or decreasing electron-phonon coupling (i.e., stiffening of the $E_{2 g}$ mode). However, the role of possible strong intraband scattering still has to be clarified and the origin of the progressive isotropization of this band with increasing $\mathrm{Al}$ doping (i.e., decrease of the upper critical field anisotropy) thus remains an open question. ${ }^{11-14}$

No $H_{c 1}$ measurements have been performed so far in Aldoped samples. The influence of doping on this field is presented in Sec. III. $H_{c 1}$ has been deduced from local Hall probe magnetization measurements for all $\mathrm{Al}$ concentrations. 
As expected from the decrease of the carrier density with doping, $H_{c 1}$ decreases with $x$ in both directions. Moreover, $\Gamma_{H_{c 1}}(0)$ remains on the order of 1 for all measured samples, indicating that the $\pi$ band remains clean in all samples. ${ }^{16}$

Finally, the evolution of the gaps with Al concentration is discussed in Sec. IV. Those gaps have been deduced from the temperature dependence of the electronic contribution of the specific heat which has been fitted to the two-gap model. Very similar values have been obtained by PCS. None of those two measurements led to the rapid decrease of $\Delta_{\pi}$ previously observed by Gonnelli et al. ${ }^{15}$ The large gap decreases roughly proportionally with $T_{c}$, never being smaller than the BCS value. The small gap is basically constant, indicating that merging of the gaps could occur below $10-15 \mathrm{~K}$. The evolution of the gaps with $\mathrm{Al}$ concentration suggests that both band filling and interband scattering effects are present.

\section{SPECIFIC HEAT MEASUREMENTS: DETERMINATION OF THE UPPER CRITICAL FIELD}

Specific heat measurements have been performed on small single crystals with $x=0,0.1$, and $\geq 0.2$ (of typical dimensions $100-200 \times 200-300 \times 20-30 \mu^{3}$ ) using an ac technique as described in Ref. 17. The magnetic fields (up to 8T) were applied both parallel and perpendicular to the $a b$ planes. The Chromel-Constantan thermocouples used to record the temperature oscillations of the samples were very carefully calibrated in situ by measuring both silicon and silver crystals of high purity. To avoid any arbitrary subtraction of the phonon contribution, in temperature sweeps the field-dependent contribution to the specific heat has been obtained by subtracting the run at $H \| c=3 \mathrm{~T}$ (i.e., above $H_{c 2}$ ) from $C_{p}(T, H): \Delta C_{p}=C_{p}(T, H)-C_{p}(T, H \| c=3 T)$. In Figs. 1 and 7 the specific heat data are presented as $C_{e l}$ $=\left(\gamma_{N} T+\Delta C_{p}\right) / \gamma_{N} T$ where the normal-state Sommerfeld coefficient $\gamma_{N}=C_{p}\left(H>H_{c 2}\right) / T_{\mid T \rightarrow 0}-C_{p}(H=0) / T_{\mid T \rightarrow 0}$ has been directly measured in field sweeps at $\sim 2 \mathrm{~K}$. For field sweeps (Fig. 4), data have been plotted as $\gamma(H) / \gamma_{N}$ with $\gamma(H)$ $=C_{p}(H) / T_{\mid T \rightarrow 0}-C_{p}(H=0) / T_{\mid T \rightarrow 0}$. These ratios are therefore measured very precisely (with no arbitrary assumptions) even though the absolute value of $C_{p}$ is not known accurately. As an independent check of the validity of our procedure, it is worth noting that the entropy conservation rule is well obeyed in Fig. 7.

Typical examples have been reported in Fig. 1; the top panel displays the evolution of the $C_{p}$ anomaly for increasing fields (for $x=0.1$ and $H \| c$ ) and the bottom panel a comparison of the zero-field and $\mu_{0} H=0.6 \mathrm{~T}$ anomalies for the three Al contents. For $x=0$ and $x=0.1$ well-defined sharp specific heat jumps were observed for all fields. The specific heat jump progressively shifts towards lower temperature for increasing field, allowing an unambiguous determination of $H_{c 2}$ (taken at the midpoint of the anomaly; see top panel of Fig. 1). For $x \gtrsim 0.2$ the zero-field anomaly is broader. However, as shown on the bottom panel of Fig. 1, those measurements still enable one determine $H_{c 2}$ precisely (down to the lowest temperature).

The $T_{c}$ values deduced from our $C_{p}$ (midpoint of the specific heat jump) for $x=0$ and $x=0.1$ are in good agreement

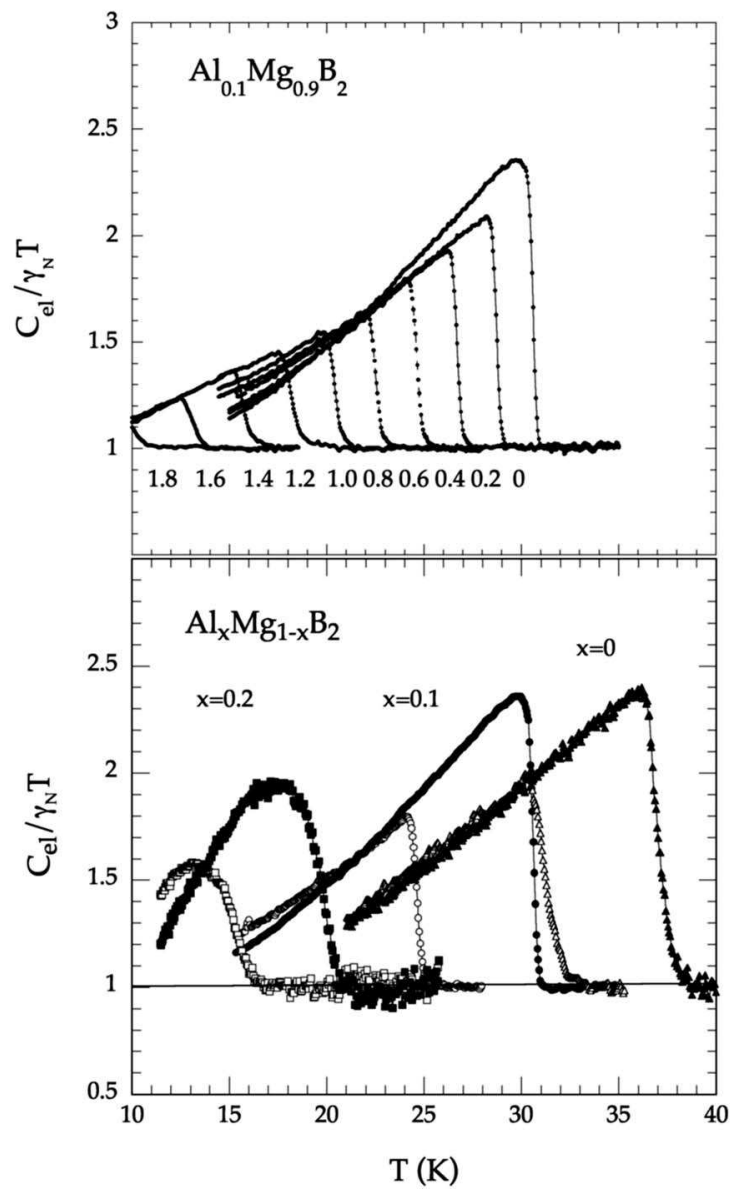

FIG. 1. Top panel: temperature dependence of the electronic contribution to the specific heat $\left(\Delta C_{p}\right)$ renormalized to the normalstate value $\left(\gamma_{N} T\right)$ in a $\mathrm{Mg}_{x} \mathrm{Al}_{1-x} \mathrm{~B}_{2}$ single crystal for $x=0.1$ at designated magnetic fields, Bottom panel: $\Delta C_{p} / \gamma_{N} T$ vs $T$ for $x=0$ (triangles), $x=0.1$ (circles), and $x=0.2$ (squares) at $H=0$ (solid symbols) and $\mu_{0} H \|_{c}=0.6 \mathrm{~T}$ (open symbols).

with previously published values (see Table I). For $x \sim 0.2$, transport measurements in single crystals by Kim et al. ${ }^{11}$ as well as in polycrystalline samples of similar composition previously led to $T_{c} \sim 23-24 \mathrm{~K}^{.13,14} \mathrm{We}$ obtained very similar values $(\sim 23 \mathrm{~K})$ from ac transmittivity measurements: $\left[B_{a c}(T)-B_{a c}\left(T \gg T_{c}\right)\right] /\left[B_{a c}\left(T \gg T_{c}\right)-B_{a c}(T \rightarrow 0)\right]$ where $B_{a c}$ is the ac field detected by a miniature Hall probe in response to an ac excitation of $\sim 5 \mathrm{G}$ at $\omega \sim 27 \mathrm{~Hz}$. However, those values are higher than those obtained by specific heat measurements [ranging from $19.5 \mathrm{~K}$ to $22.5 \mathrm{~K}$ in samples (a) and (b), respectively]. This suggests that the $\mathrm{Al}$ content in the bulk is probably slightly higher than the one of the surface. Indeed, a small fraction of lower $\mathrm{Al}$ content on the surface of the sample will not show up in the specific heat signal but may lead to some diamagnetic screening (and corresponding drop of the resistivity)..$^{18}$ Note that this inhomogeneity remains at the surface since (bulk) specific heat measurements are very sensitive to sample inhomogeneities and all samples presented here did unambiguously show a jump with a typical width on the order of $2 \mathrm{~K}$. A typical example has been reported in Fig. 1(b) $\left(T_{c}=19.5 \mathrm{~K}\right.$ sample). A lower value $(\sim 21 \mathrm{~K})$ has been obtained from PCS measurements in 
TABLE I. $x$ is the $\mathrm{Al}$ content in $\mathrm{Mg}_{1-x} \mathrm{Al}_{x} \mathrm{~B}_{2}$ single crystals. The upper critical field $H_{c 2}$ has been deduced from the midpoint of the specific heat anomaly and the lower critical field $H_{c 1}$ from local magnetization measurements. The gaps values have been determined from the temperature dependence of the specific heat $\left(\Delta_{\pi}^{C_{p}}\right.$ and $\left.\Delta_{\sigma}^{C_{p}}\right)$ and/or from point contact spectroscopy measurements $\left(\Delta_{\pi}^{P C S}\right.$ and $\left.\Delta_{\sigma}^{P C S}\right)$. Critical fields and gap values are given for $T \rightarrow 0$ and $T_{c}$ has been deduced from $C_{p}$ measurements (respectively, PCS).

\begin{tabular}{|c|c|c|c|c|c|c|c|c|c|}
\hline$x$ & $H_{c 2}^{c}(T)$ & $H_{c 2}^{a b}(T)$ & $H_{c 1}^{c}(G)$ & $H_{c 1}^{a b}(G)$ & $T_{c}(\mathrm{~K})$ & $\Delta_{\sigma}^{C_{p}}(\mathrm{meV})$ & $\Delta_{\pi}^{C_{p}}(\mathrm{meV})$ & $\Delta_{\sigma}^{P C S}(\mathrm{meV})$ & $\Delta_{\pi}^{P C S}(\mathrm{meV})$ \\
\hline 0 & $3.0 \pm 0.3$ & $16.0 \pm 0.5$ & $1100 \pm 100$ & $1100 \pm 100$ & $36-37$ & $7.1 \pm 0.4$ & $2.4 \pm 0.4$ & $6.7 \pm 0.4$ & $2.3 \pm 0.2$ \\
\hline 0.1 & $2.6 \pm 0.2$ & $7.3 \pm 0.3$ & $820 \pm 100$ & $800 \pm 100$ & 31 & $5.6 \pm 0.4$ & $2.6 \pm 0.4$ & $5.1 \pm 0.4$ & $2.3 \pm 0.2$ \\
\hline$\gtrsim 0.2$ (a) & $1.8 \pm 0.2$ & $2.8 \pm 0.2$ & $500 \pm 100$ & $520 \pm 100$ & 19.5 & $3.3 \pm 0.5$ & $2.3 \pm 0.5$ & & \\
\hline$\gtrsim 0.2$ (b) & $1.9 \pm 0.2$ & $3.9 \pm 0.2$ & & & 22.5 (resp. 21) & $3.5 \pm 0.3$ & $1.9 \pm 0.3$ & $3.4 \pm 0.4$ & $1.8 \pm 0.2$ \\
\hline
\end{tabular}

sample (b) probably related to the presence of a minority phase at the surface of this sample as also seen in transmittivity measurements. As the composition is directly related to the $T_{c}$ value, all results will thus be presented as a function of $T_{c}$ (and not nominal $x$ value).

The $H_{c 2}$ values deduced from our specific heat measurements have been reported in Fig. 2(a). In the undoped sample, $H_{c 2}^{a b}$ exceeds $8 \mathrm{~T}$ for $T<20 \mathrm{~K}$ and the lowtemperature values were deduced from high field magnetotransport data. ${ }^{3}$ Very similar $H_{c 2}(0)$ values have been obtained in all $x \gtrsim 0.2$ samples. The $H_{c 2}$ values corresponding to the onset of diamagnetic screening have also been reported in Fig. 2 (for $x \gtrsim 0.2$, dotted lines). This criterion leads to a small upward curvature close to $H=0$ in both directions. A similar curvature has been obtained by Kim et al. ${ }^{11}$ from transport measurements and has been interpreted as being consistent with the dirty-limit two-gap theory. However, as this curvature has not been confirmed by specific heat measurements, it is most probably related to (surface) sample inhomogeneities. No difference between specific heat and magnetic measurements could be observed for $x=0$ and $x=0.1$.

Figure 2(b) displays our $H_{c 2}(T \rightarrow 0)$ values deduced from specific heat measurements (midpoint of the transition) as a function of $T_{c}$ (circles) together with the values previously obtained by Angst et al. ${ }^{14}$ (squares) and Kim et al. ${ }^{11}$ (triangles). As shown all measurements agree on a substantial decrease of $H_{c 2}^{a b}$ with $\mathrm{Al}$ content even though our specific heat measurements lead to a value significantly lower than the previously published ones for $x=0.1$. As observed by Kim et al., ${ }^{11} H_{c 2}^{c}$ also slightly decreases with $T_{c}$.

In magnesium diboride, the $H_{c 2}(0)$ values are mainly determined by the parameters of the $\sigma$ band due to the rapid suppression of superconductivity in the $\pi$ band at high field. As discussed in Refs. 13 and 14, in the clean limit $H_{c 2}(0)$ values are given by

$$
\begin{aligned}
& \mu_{0} H_{c 2}^{a b}(0)=\frac{\Phi_{0} \pi \Delta_{\sigma}^{2}}{2 \hbar^{2} v_{F}^{a b, \sigma} v_{F}^{c, \sigma}}, \\
& \mu_{0} H_{c 2}^{c}(0)=\frac{\Phi_{0} \pi \Delta_{\sigma}^{2}}{2 \hbar^{2}\left(v_{F}^{a b, \sigma}\right)^{2}},
\end{aligned}
$$

where $v_{F}^{a b, \sigma}$ and $v_{F}^{c, \sigma}$ are the Fermi velocities in the corresponding directions. The decrease of both $H_{c 2}(0)$ values can then be, at least qualitatively, attributed to the decrease of the superconducting gap $\Delta_{\sigma}$ due to electronic doping and/or stiffening of the $E_{2 g}$ phonon mode. For $H \| c$, this decrease is partially compensated by a decrease of the in-plane Fermi velocity $v_{F}^{a b, \sigma}\left(v_{F}^{c, \sigma}\right.$ remaining approximatively constant $\left.{ }^{13}\right)$, thus leading to an only slightly decreasing $H_{c 2}^{c}$ value (see Fig. 2 and Table I). The corresponding Fermi velocities can then be deduced from the $H_{c 2}$ and $\Delta_{\sigma}$ (see below and Table I) values. We hence get $v_{F}^{a b, \sigma} \approx 3.5,3.0$, and $2.0 \times 10^{5} \mathrm{~m} / \mathrm{s}$ and $v_{F}^{c, \sigma} \approx 0.7,0.9$, and $1.3 \times 10^{5} \mathrm{~m} / \mathrm{s}$ for $x=0,0.1$, and $\gtrsim 0.2$, respectively. Those values are in reasonable agreement with those calculated by Putti et al. ${ }^{13}$ from the electronic structure at various $\mathrm{Al}$ contents even though they are slightly shifted.

The temperature dependence of $\Gamma_{H_{c 2}}$ is displayed in Fig. 3 . Those values are in good agreement with those previously reported by Angst et al. ${ }^{14}$ and Kim et al. ${ }^{11}$ Note that $\Gamma_{H_{c 2}} \sim 1.5$ is almost temperature independent for $x \gtrsim 0.2$ $\left(T_{c}^{c 2}=19.5 \mathrm{~K}\right.$ sample; similarly we obtained an almost temperature-independent value $\sim 1.6$ from transmittivity measurements). In the clean limit $\Gamma_{H_{c 2}}(0)$ is equal to the ratio between the Fermi velocities, $v_{F}^{a b, \sigma} / v_{F}^{c, \sigma}$, and the rapid decrease of $v_{F}^{a b}$ associated with an almost constant $v_{F}^{c}$ value thus naturally accounts for the fast decrease of the upper critical field anisotropy. However, this decrease is larger than the calculated one [following Ref. $13 \Gamma_{H_{c 2}}(0)$ should be on the order of 4 for $x=0.2$ ] and, as suggested by Kim et al., ${ }^{11}$ it could originate not only from the change in the Fermi velocities due to electronic doping but also from an anisotropic increase of impurity scattering.

Figure 4 displays the magnetic field dependence of the Sommerfeld coefficient $\gamma=\Delta C_{p} /\left.T\right|_{T \rightarrow 0}$ for $x=0,0.1$, and $\gtrsim 0.2\left(T_{c}=22.5 \mathrm{~K}\right.$ sample) for $H \| c$ and $T=2.5 \mathrm{~K}$. As previously observed by Bouquet et al., ${ }^{19}$ the $\gamma$ vs $H$ curve is strongly nonlinear and a kink is visible for $H / H_{c 2}=h_{k i n k}$ $\gtrsim 0.2$. In classical systems, $\gamma \propto\left[\xi / a_{0}\right]^{2}$ where $a_{0}$ is the vortex spacing and a curvature has been recently predicted by Kogan and Zhelezina ${ }^{21}$ in the clean limit due to a shrinkening of the core size $[\xi(H)]$. However, the nonlinearity observed for $H \| a b$ (Ref. 19) is muchsteeper than the calculated one ( $\gamma$ reaches $50 \%$ of its normal-state value for $h \sim 1 / 20$ ), clearly emphasizing the role of the two-gap nature of $\mathrm{MgB}_{2}$. As discussed in Ref. 19, this behavior can then be qualitatively attributed to the rapid filling of the small gap with field. For the $T_{c}=19.5 \mathrm{~K}$ sample, our measurements rather suggest a linear field dependence of $\gamma$ as expected for classical one gap (dirty) superconductors for which $\gamma \propto\left[\xi / a_{0}\right]^{2}$ $\propto H / H_{c 2}$. However, the very small value of the specific heat 

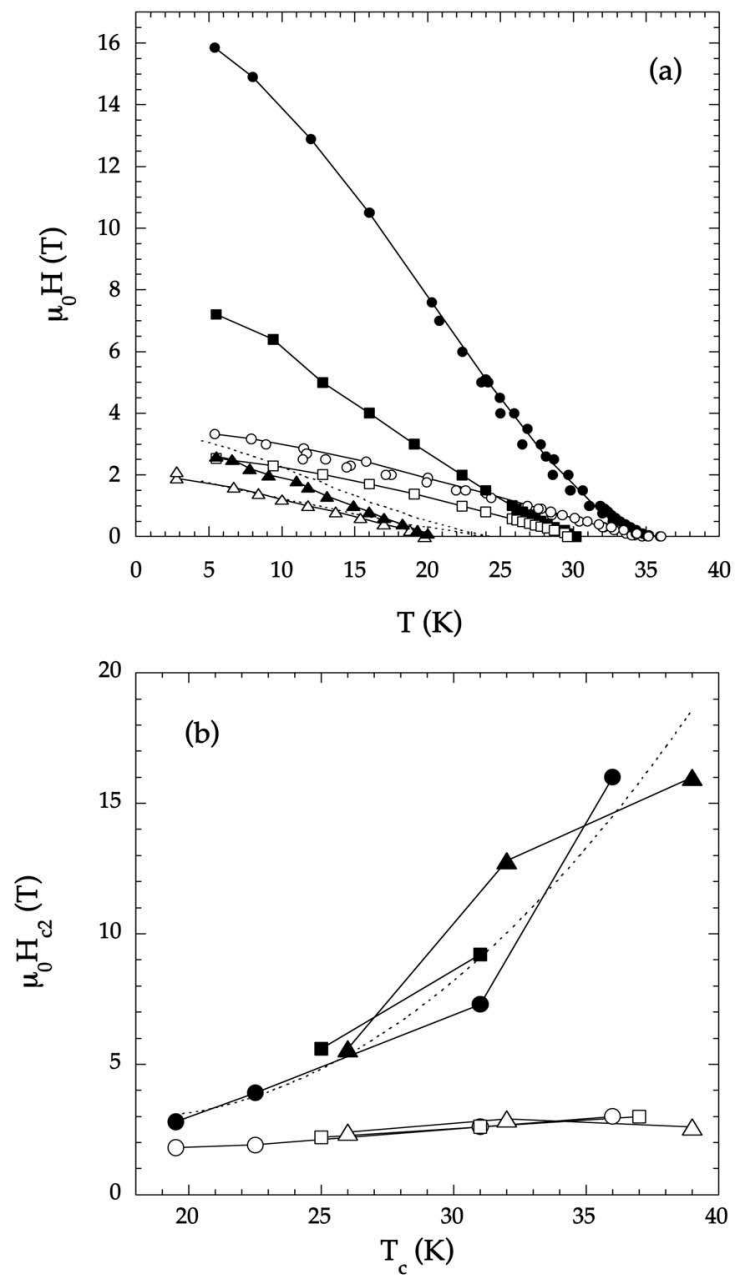

FIG. 2. (a) Temperature dependence of the $H_{c 2}^{c}$ (open symbols) and $H_{c 2}^{a b}$ (solid symbols) deduced from specific heat measurements (midpoint of the $C_{p}$ anomaly; see 1) in $\mathrm{Mg}_{1-x} \mathrm{Al}_{x} \mathrm{~B}_{2}$ single crystals for $x=0$ (circles), $x=0.1$ (squares), and $x=0.2$ (triangles). The lowtemperature data for $x=0, H \| a b$ have been deduced from magnetotransport measurements (Ref. 3). The dotted lines correspond to the $H_{c 2}$ values deduced from the onset of diamagnetic response in $x=0.2$. (b) $H_{c 2}$ as a function of the critical temperature (the dotted line is a guide to the eyes) for $H \|_{c}$ (open symbols) and $H \| a b$ (solid symbols) from this work (circles), Ref. 11 (triangles), and Ref. 14 (squares).

jump in this latter case did not enable us to completely exclude the presence of some small nonlinearity.

Assuming that the kink hence corresponds to the filling of the small gap $\left(H_{\text {kink }} \sim H_{c 2}^{\pi}\right)$, one obtains the same $H_{c 2}^{\pi} \sim 0.5-1 \mathrm{~T}$ for all samples. If $H_{c 2}^{\pi} \propto\left[\Delta_{\pi} / v_{F}^{a b, \pi}\right]^{2}$, this would suggest that $v_{F}^{a b, \pi}$ remains approximatively unchanged $\left[\Delta_{\pi}(x=0.1) \approx \Delta_{\pi}(x=0)\right.$; see below]. However, it is important to note that Zhitomirsky and Dao ${ }^{20}$ suggested that, at low field, the vortex core size $\left[\xi_{v}(0)=\max \left(\xi_{\pi}, \xi_{\sigma}\right) \sim \xi_{\pi}\right]$ might actually not be given by the parameters of the $\pi$ band-i.e., that $\xi_{\pi} \neq \hbar v_{F}^{a b, \pi} / \pi \Delta_{\pi}$-but would be on the order of 1-2 $\times \xi_{\sigma}$. This directly leads to $h_{\text {kink }} \sim\left[\xi_{\sigma} / \xi_{v}(0)\right]^{2} \sim 1-1 / 4$ in reasonable agreement with the experimental value $\gtrsim 0.2$.

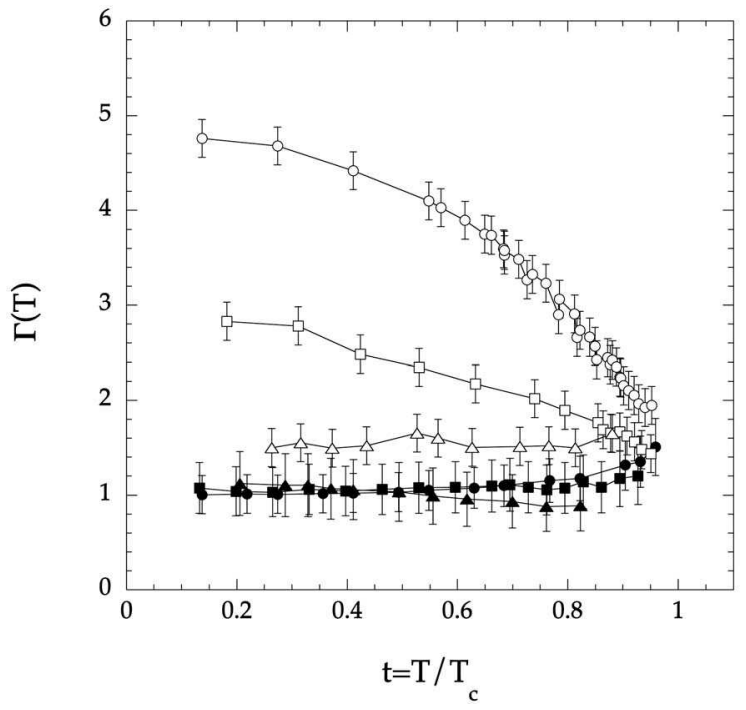

FIG. 3. Temperature dependence of the anisotropy of the upper (open symbols) and lower (solid symbols) critical fields in $\mathrm{Mg}_{1-x} \mathrm{Al}_{x} \mathrm{~B}_{2}$ single crystals for $x=0$ (circles), $x=0.1$ (squares), and $x=0.2$ (triangles).

\section{HALL PROBE MAGNETOMETRY: DETERMINATION OF THE LOWER CRITICAL FIELD}

The stray field at the surface of the sample has been measured locally using miniature Hall probe arrays for various applied dc fields. The first penetration field $\left(H_{p}\right)$ has been defined as the field for which a nonzero magnetic field is detected by the Hall probe located close to the center of the sample (see Fig. 5 for $H \|_{c}$; this value is not affected by the position of the probe due the absence of significant bulk pinning). Note that the flux entry is much sharper for $x=0$ and $x=0.1$ than for $x \gtrsim 0.2$, again reflecting the presence of some (surface) inhomogeneities in the latter case.

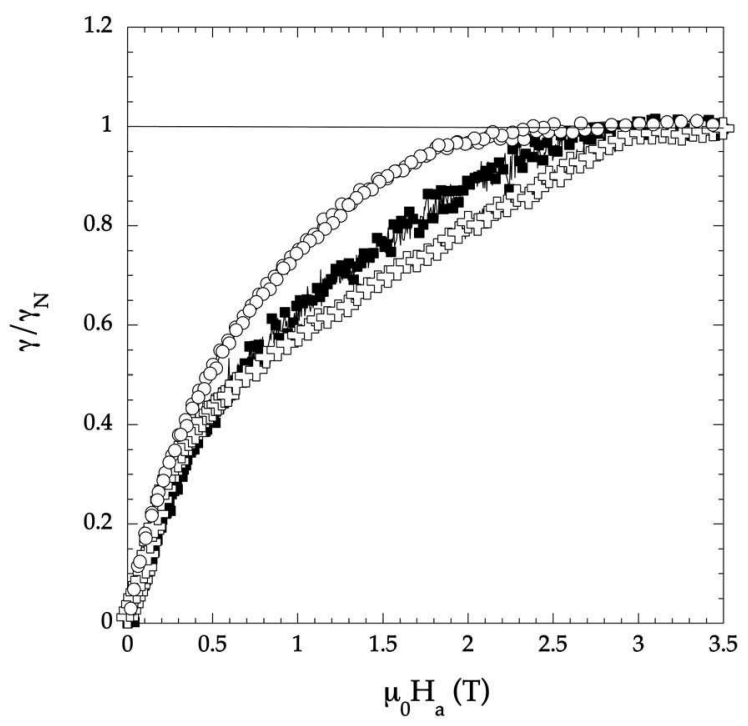

FIG. 4. Magnetic field dependence of the Sommerfeld coefficient (at $T=2.5 \mathrm{~K}, H \| c$ ) in $\mathrm{Mg}_{1-x} \mathrm{Al}_{x} \mathrm{~B}_{2}$ single crystals for $x=0$ (crosses), $x=0.1$ (squares), and $x=0.2\left(T_{c}=22.5 \mathrm{~K}\right.$ sample, circles). 


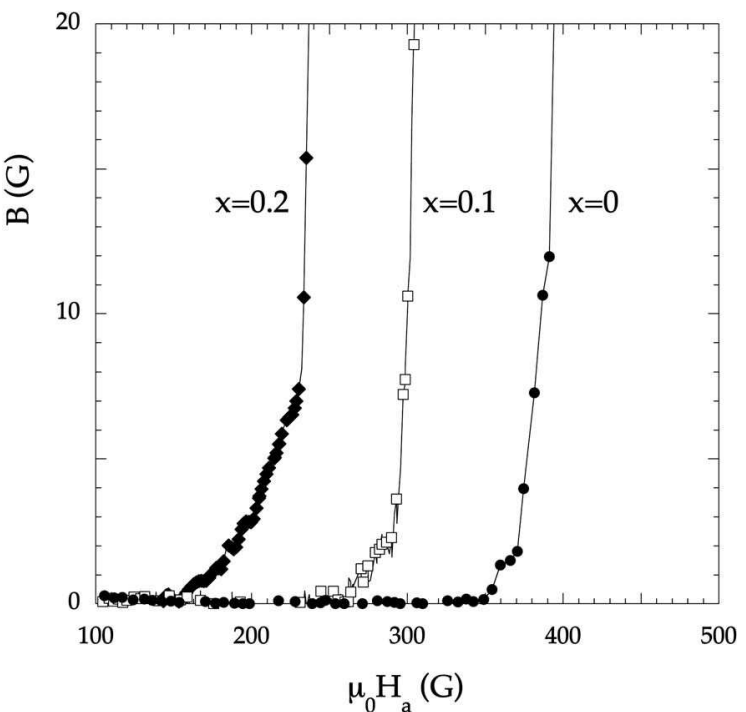

FIG. 5. Induction $(B)$ at the center of the sample detected by a miniature Hall probe as a function of the applied field $\left(H_{a} \| c\right)$ (at $T=2.5 \mathrm{~K}, H \| c$ ) in $\mathrm{Mg}_{1-x} \mathrm{Al}_{x} \mathrm{~B}_{2}$ single crystals for $x=0$ (circles), $x$ $=0.1$ (squares), and $x=0.2$ (diamonds). A finite induction is detected for $H_{a}>H_{p}$.

As shown in Ref. 7 geometrical barriers play a dominant role in the vortex penetration process for $H \| c$ and $H_{c 1}$ is then related to $H_{p}$ through $^{22}$

$$
H_{c 1}=\frac{H_{p}}{\tanh \sqrt{\alpha \frac{d}{2 w}}},
$$

where $\alpha$ is a numerical constant related to the sample geometry and $d$ and $2 w$ are the thickness and width of the sample, respectively. $H_{c 1}$ has thus been precisely deduced from $H_{p}$ in the pure sample by measuring a collection of samples of very different $d / 2 w$ ratios. We hence got $H_{c 1}^{c} \sim 1100 \mathrm{G}$ in the pure sample $^{7}$ and, by comparison to the undoped sample of same $d / 2 w$ ratio, $H_{c 1}^{c} \sim 800 \mathrm{G}$ and $H_{c 1}^{c} \sim 500 \mathrm{G}$ for $x=0.1$ and $x$ $\gtrsim 0.2$, respectively. For $H \| a b$, the influence of geometrical barriers can be neglected and $H_{c 1}^{a b} \sim H_{p}^{a b} /\left(1-N_{a b}\right)$ where $N_{a b}$ is the geometrical coefficient of the corresponding sample. We hence got $H_{c 1}^{a b}=1100,800$, and $520 \mathrm{G}$ for $x=0,0.1$, and $\gtrsim 0.2$, respectively (see Table I).

The temperature dependence of the corresponding $H_{c 1}$ values is displayed in Fig. 6 for all three samples. As shown $H_{c 1}(0)$ decreases with increasing $x$ for both directions, reflecting the decrease of the superfluid density as the $\sigma$ band is progressively filled up by electronic doping. However, the anisotropy parameter remains on the order of 1 in all three samples (see Fig. 3). In the clean limit, the anisotropy of $\lambda$ (which is on the order of $\Gamma_{H_{c 1}}$; see below) is related to the average of the squared Fermi velocity over the entire Fermi surface (i.e., taking into account both bands). ${ }^{16}$ Our measurements thus suggest that this average is independent of doping. Note that a significant increase of the dirtiness of the $\pi$ band would have led to an increase of this anisotropy. ${ }^{16}$

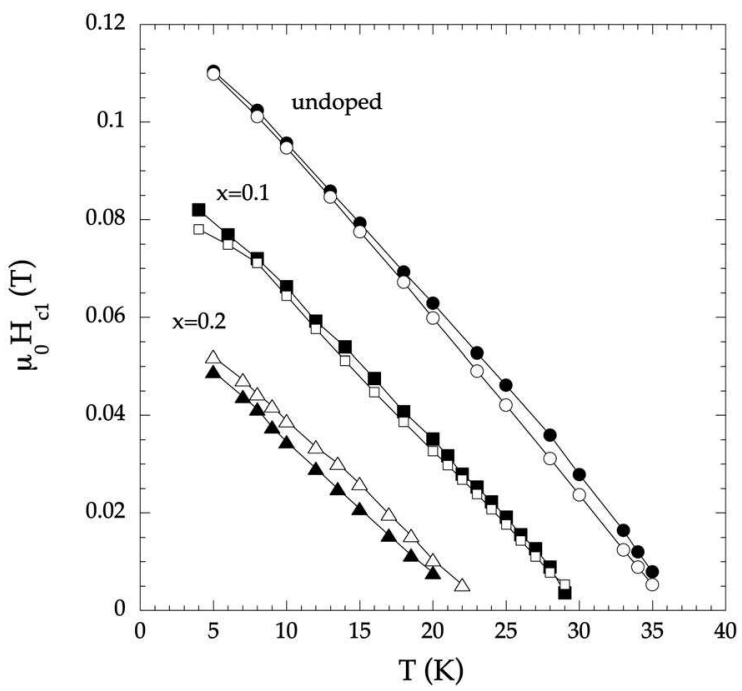

FIG. 6. Temperature dependence of the $H_{c 1}^{c}$ (solid symbols) and $H_{c 1}^{a b}$ (open symbols) deduced from Hall probe magnetometry measurements (see Fig. 5) taking into account the presence of geometrical barriers for $H \| c$ (see text for details) in $\operatorname{Mg}_{1-x} \mathrm{Al}_{x} \mathrm{~B}_{2}$ single crystals for $x=0$ (circles), $x=0.1$ (squares), and $x=0.2$ (triangles).

The lower critical field is related to the penetration depth through $^{23}$

$$
\begin{gathered}
\mu_{0} H_{c 1}^{a b}=\frac{\Phi_{0}}{4 \pi \lambda_{a b} \lambda_{c}}\left[\ln \left(\kappa_{b}\right)+\alpha\left(\kappa_{b}\right)\right], \\
\mu_{0} H_{c 1}^{c}=\frac{\Phi_{0}}{4 \pi \lambda_{a b}^{2}}\left[\ln \left(\kappa_{c}\right)+\alpha\left(\kappa_{c}\right)\right],
\end{gathered}
$$

where $\kappa_{b}=\lambda_{c} / \xi_{a}=\lambda_{c} / \lambda_{a} \times \lambda_{a} / \xi_{a}=\Gamma_{\lambda} \times \kappa_{c}$ and $\alpha(\kappa)=0.5$ $+1.693 /(2 \kappa+0.586) .{ }^{24}$ However, deducing $\lambda$ from $H_{c 1}$ remains difficult in magnesium diboride as $\kappa_{b}$ and $\kappa_{c}$ are field dependent. A "low field" $\xi_{a b}$ value, different from $\sqrt{\Phi_{0} / 2 \pi B_{c 2}^{c}}$, has to be used, leading to a $\kappa_{c}$ value ranging from 2 to 3 at low field to $\sim 7$ close to $H_{c 2}$ and $\lambda_{a b}$ increasing from $\sim 450-500 \AA$ to $\sim 700-800 \AA .{ }^{25}$ However, for $x$ $\gtrsim 0.2$ the system can be consistently described as a classical one-gap superconductor with $\lambda_{a b} \sim 850 \AA, \xi_{a b} \sim 130 \AA, \Gamma_{\lambda}$ $=\Gamma_{\xi}=\Gamma_{H_{c 2}} \sim 1.5$, and, correspondingly, $\Gamma_{H_{c 1}} \sim 1.3$ which falls into the error bars of our measurements. Note that this $\lambda_{a b}$ value (and $T_{c}$ value) is very close to the one obtained for the $\pi$ band in pure $\mathrm{MgB}_{2}$ by Zehetmayer et al. ${ }^{25}$ again suggesting that the $\sigma$ band has been almost completely filled up by doping.

\section{INFLUENCE OF AI DOPING ON THE SUPERCONDUCTING GAPS}

Figure 7 displays the temperature dependence of the zerofield electronic contribution to the specific heat $\left(\Delta C_{p}\right.$, renormalized to the normal-state value $\gamma_{N} T$ ) for the three Al contents (the curves have been vertically shifted upwards for clarity in the doped samples). As shown, a clear deviation from the standard BCS dependence is observed for $x=0$ (dotted line; a similar deviation is observed for $x=0.1$, not 


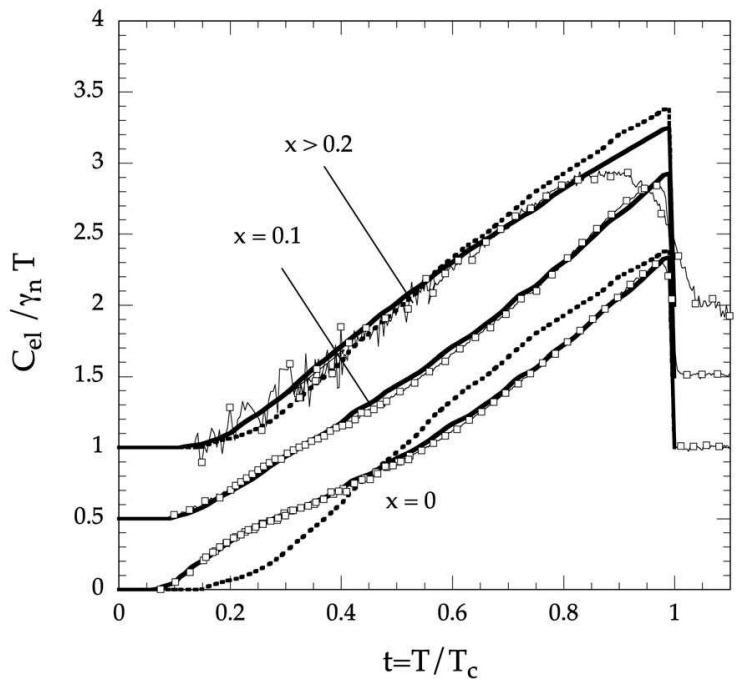

FIG. 7. Temperature dependence of the zero-field electronic contribution to the specific heat $\left(\Delta C_{p}\right.$, renormalized to the normalstate value $\left.\gamma_{N} T\right)$ in $\mathrm{Mg}_{1-x} \mathrm{Al}_{x} \mathrm{~B}_{2}$ single crystals for $x=0, x=0.1$, and $x \gtrsim 0.2\left(T_{c}=19.5 \mathrm{~K}\right.$ sample); the curves have been arbitrarily shifted for clarity. The solid lines are fits to the data assuming that the contribution of each band can be added separately. The dotted lines are the BCS curves (not shown for $x=0.1$ ).

shown). An excess of specific heat is observed at low $T$ due to the presence of the small gap which is compensated by a reduced $C_{p}$ above $t \sim 0.4$. Following Ref. 26, those curves have been fitted to the two-band theory in order to obtain the gap values (solid lines). The total specific heat is here considered to be the sum of the contribution of each band with a relative weight $\omega_{\sigma}$ and $\omega_{\pi}=1-\omega_{\sigma}$, respectively. The experimental curves have thus been adjusted to the model with three parameters $\Delta_{\sigma}, \Delta_{\pi}$, and $\omega_{\sigma}$. As previously pointed out in polycrystals by Putti et al. ${ }^{13}$ we did not observe any significant change of $\omega_{\sigma}$ for $x=0$ and $x=0.1$. The partial contribution of each band remains close to 0.5 for both samples in good agreement with calculations by Liu et al. ${ }^{27}$ in pristine samples.

As the gap values are getting very close for $x \geqslant 0.2$ the uncertainty on $\omega_{\sigma}$ is getting large in this case $\left(T_{c}=19.5 \mathrm{~K}\right.$ sample) and a standard BCS dependence (which would correspond to the presence of only one merged gap) cannot be completely excluded even though the "best fit" leads to $\Delta_{\sigma} \sim 3.3 \mathrm{meV}$ and $\Delta_{\pi} \sim 2.3 \mathrm{meV}$ (with $\omega_{\sigma} \sim 0.4$ ).

Point contact spectroscopy measurements have been performed in samples of a similar batch with $T_{c}$ of $37 \mathrm{~K}, 31 \mathrm{~K}$, and $21 \mathrm{~K}$ deduced from PCS measurements (closing of the gaps). A standard lock-in technique has been used to measure the differential resistance as a function of the voltage applied on the contacts. As a direct transfer of carriers with energy $e V<\Delta$ is forbidden, Andreev reflection of a hole back into the normal metal wires (associated with the formation of a Cooper pair in the superconductor) leads to a 2 times higher conductance. However, due to the incomplete transmission of the contact, a dip is observed in the conductance spectra which can then be fitted using the Blonder-TinkhamKlapwijk (BTK) theory ${ }^{28}$ using the gap values, partial contributions of each band, transparency, and quasiparticle broadening as parameters.

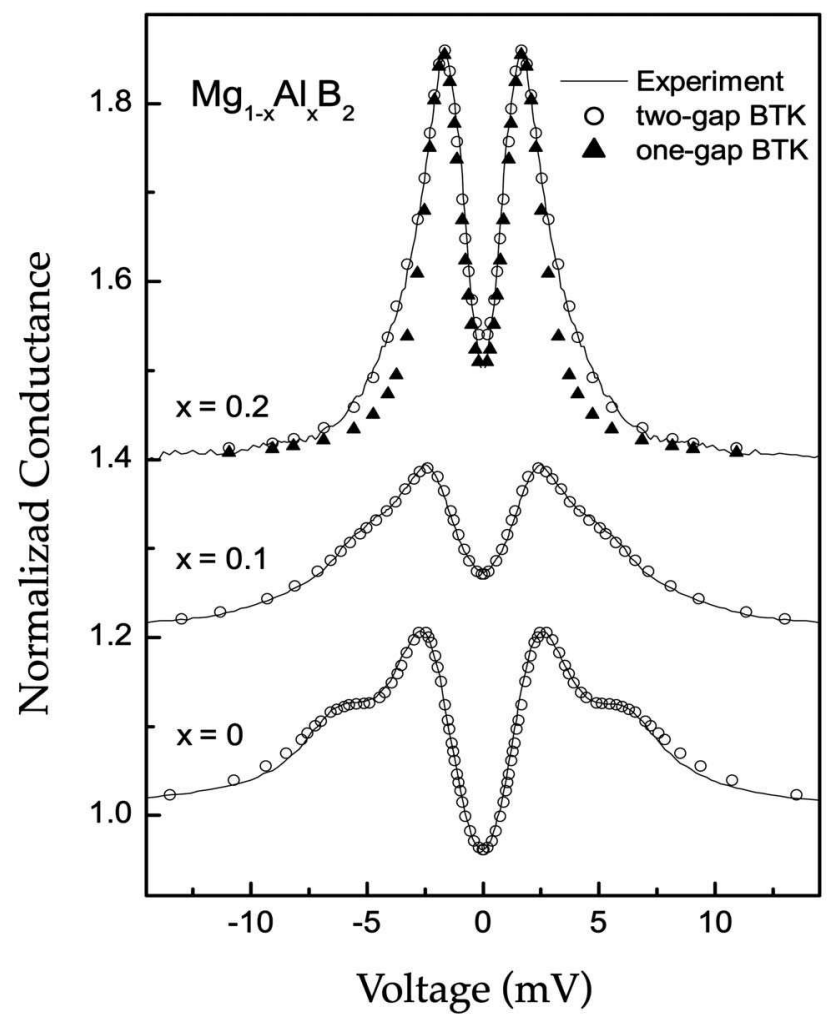

FIG. 8. Typical example of a point contact spectrum obtained for $x=0,0.1$, and 0.2 (solid lines) and corresponding BTK fits for two- gap superconductors (open circles; see text for details). The solid triangles correspond to a BTK fit in a one-gap model. The curves have been arbitrarily shifted for clarity.

Figure 8 displays typical examples of the normalized conductance versus voltage spectra for all three $\mathrm{Al}$ concentrations. At $x=0$ and 0.1 the spectra clearly reveal the two-gap structure in the form of symmetrically placed peaks (humps at the voltage position of $\Delta_{\sigma}$ for $x=0.1$ ). For the highest $\mathrm{Al}$ concentration only a single pair of peaks is seen but the spectrum cannot be fitted by the single-gap BTK conductance (solid triangles). On the contrary, all spectra can be well fitted by the two-gap BTK model (open circles), yielding the large and small gaps as indicated in Table I. The close position of the two gaps prevents better resolution of the large gap in the point contact spectrum, even with a relatively high contribution of the $\sigma$ band which was about $20 \%$ in the presented case. We also remark that the single-gap fit necessarily leads to a small gap value with the coupling ratio $2 \Delta / k T_{c}$ much smaller than the canonical BCS value, giving yet another piece of evidence that two gaps are still retained at this $\mathrm{Al}$ concentration. The corresponding gaps are in good agreement with our specific heat measurements. All values have been reported in Fig. 9; squares correspond to the specific heat data whereas circles were obtained from PCS. We have also reported the gap values previously obtained by Putti et al. ${ }^{13}$ from specific heat measurements in polycrystals and Gonnelli et al. ${ }^{15}$ from spectroscopic measurements in single crystals.

As shown in Fig. 9, $\Delta_{\sigma}$ decreases almost linearly with the critical temperature whereas $\Delta_{\pi}$ remains approximatively 


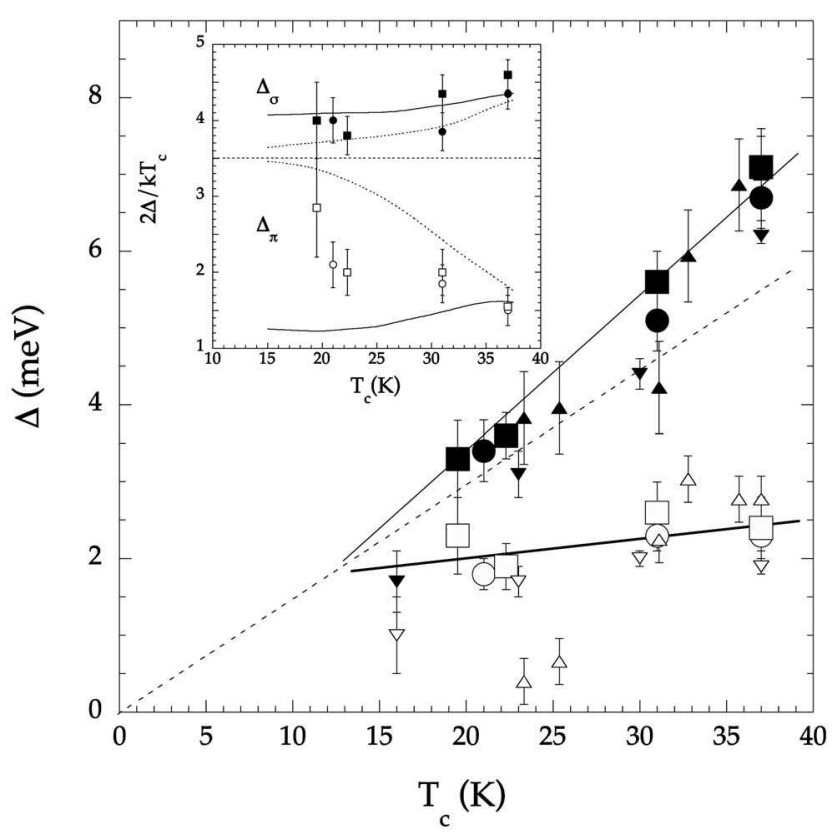

FIG. 9. Small- and large-gap values as a function of the critical temperature in $\mathrm{Mg}_{1-x} \mathrm{Al}_{x} \mathrm{~B}_{2}$ single crystals deduced from specific heat (squares) and point contact spectroscopy (circles) measurements. The values previously obtained from spectroscopy measurements in single crystals by Gonnelli et al. (Ref. 11) (upwards triangles) and specific heat measurements in polycrystals by Putti et al. (Ref. 9) (downwards triangles) have also been reported. The dotted line corresponds to the BCS prediction $\Delta=1.76 k T_{c}$. The solid lines are guides to the eyes. In the inset: $2 \Delta / k T_{c}$ as a function of the critical temperature in $\mathrm{Mg}_{1-x} \mathrm{Al}_{x} \mathrm{~B}_{2}$ single crystals deduced from specific heat (squares) and point contact spectroscopy (circles) measurements. Calculations by Kortus et al. (Ref. 29) without interband scattering (solid line) and for $\gamma_{\text {inter }}=2000 \mathrm{x} \mathrm{cm}^{-1}$ are also reported.

constant. We did not observe the rapid drop of $\Delta_{\pi}$ above $x$ $\sim 0.1$ reported by Gonnelli et al. ${ }^{15}$ Our large gap values are also larger than those obtained by Putti et al., ${ }^{13}$ and the corresponding $2 \Delta_{\sigma} / k T_{c}$ ratio remains larger than the BCS 3.52 value for all samples. Indeed, as shown in the inset of Fig. 9, this ratio slightly decreases with increasing doping whereas $2 \Delta_{\pi} / k T_{c}$ increases from $\sim 1.5$ in undoped samples towards $\sim 2.5$ for $x>0.2$ (in $C_{p}$ measurements). Such an increase can be attributed to an increase of interband scattering with $\mathrm{Al}$ content. Indeed, electronic doping alone would lead to almost $x$-independent $2 \Delta / k T_{c}$ ratios (see the solid line in the inset of Fig. 9) whereas interband scattering leads to a progressive merging of the gaps. As an example we have reported in the inset of Fig. 9 (dotted lines) the evolution of the gaps calculated by Kortus et al. ${ }^{29}$ solving the Eliashberg equations in the presence of interband scattering. Obviously, the corresponding scattering term (assumed to be proportional to the doping content: $\gamma_{\text {inter }}=2000 x \mathrm{~cm}^{-1}, x$ being the Al content) is too large to reproduce the experimental data but our data clearly suggest that interband scattering is increasing with doping in Al-doped samples and could lead to a merging of the gaps around $10-15 \mathrm{~K}$.

\section{CONCLUSION}

We measured the changes of critical fields and gap values due to $\mathrm{Al}$ doping in $\mathrm{Mg}_{1-x} \mathrm{Al}_{x} \mathrm{~B}_{2}$ single crystals by specific heat measurements, Hall probe magnetometry, and point contact spectroscopy measurements. We have shown that the upper and lower critical fields both parallel and perpendicular to the $a b$ planes decrease with increasing doping mainly due to electronic doping and/or stiffening of the $E_{2 g}$ phonon mode. In contrast to carbon doping for which a significant increase of impurity scattering leads to an increase of the $H_{c 2}$ values, the role of intraband scattering in $(\mathrm{Mg}, \mathrm{Al}) \mathrm{B}_{2}$ still has to be clarified, but the evolution of the gaps clearly suggests that interband scattering increases with $\mathrm{Al}$ content. Both critical fields and gap measurements suggest that the two gaps are close to merge for $T_{c} \sim 10-15 \mathrm{~K}$ (i.e., $x \geqslant 0.3$ ).

\section{ACKNOWLEDGMENTS}

This work has been supported by the Slovak Science and Technology Assistance Agency under Contract No. APVT51-016604. The Centre of Low Temperature Physics is operated as the Centre of Excellence of the Slovak Academy of Sciences under Contract No. I/2/2003.
${ }^{1}$ P. Szabó, P. Samuely, J. Kačmarčík, T. Klein, J. Marcus, D. Fruchart, S. Miraglia, C. Marcenat, and A. G. M. Jansen, Phys. Rev. Lett. 87, 137005 (2001); P. Samuely, P. Szabó, J. Kačmarčík, T. Klein, and A. G. M. Jansen, Physica C 385, 244 (2003).

${ }^{2}$ F. Bouquet, R. A. Fisher, N. E. Philips, D. G. Hinks, and J. D. Jorgensen, Phys. Rev. Lett. 87, 047001 (2001).

${ }^{3}$ L. Lyard, P. Samuely, P. Szabó, T. Klein, C. Marcenat, L. Paulius, K. H. P. Kim, C. U. Jung, H.-S. Lee, B. Kang, S. Choi, S.-I. Lee, J. Marcus, S. Blanchard, A. G. M. Jansen, U. Welp, G. Karapetrov, and W. K. Kwok, Phys. Rev. B 66, 180502(R) (2002).

${ }^{4}$ S. L. Bud'ko, V. G. Kogan, and P. C. Canfield, Phys. Rev. B 64, 180506(R) (2001).

${ }^{5}$ M. Angst, R. Puzniak, A. Wisniewski, J. Jun, S. M. Kazakov, J.
Karpinski, J. Roos, and H. Keller, Phys. Rev. Lett. 88, 167004 (2002).

${ }^{6}$ U. Welp, A. Rydh, G. Karapetrov, W. K. Kwok, G. W. Crabtree, Ch. Marcenat, L. Paulius, T. Klein, J. Marcus, K. H. P. Kim, C. U. Jung, H-S. Lee, B. Kang, and S-I. Lee, Phys. Rev. B 67, 012505 (2003)

${ }^{7}$ L. Lyard, P. Szabó, T. Klein, J. Marcus, C. Marcenat, K. H. Kim, B. W. Kang, H. S. Lee, and S. I. Lee, Phys. Rev. Lett. 92, 057001 (2004); L. Lyard, T. Klein, J. Marcus, R. Brusetti, C. Marcenat, M. Konczykowski, V. Mosser, K-H. Kim, B. W. Kang, H-S. Lee, and S-I. Lee, Phys. Rev. B 70, 180504(R) (2004).

${ }^{8}$ R. R. Ribeiro, S. L. Budko, C. Petrovic, and P. C. Canfield, Physica C 384, 227 (2003). 
${ }^{9}$ Z. Hol'anová, J. Kačmarčík, P. Szabó, P. Samuely, I. Sheikin, R. A. Ribeiro, S. L. Bud'ko, and P. C. Canfield, Physica C 404, 195 (2004).

${ }^{10}$ R. H. T. Wilke, S. L. Bud'ko, P. C. Canfield, D. K. Finnemore, R. J. Suplinskas, and S. T. Hannahs, Phys. Rev. Lett. 92, 217003 (2004).

${ }^{11}$ H-J. Kim, H.-S. Lee, B. Kang, W-H. Yim, Y. Jo, M-H. Jung, and S-I. Lee, cond-mat/0512242 (unpublished).

${ }^{12}$ M. Putti, M. Affronte, P. Manfrinetti, and A. Palenzona, Phys. Rev. B 68, 094514 (2003).

${ }^{13}$ M. Putti, C. Ferdeghini, M. Monni, I. Pallecchi, C. Tarantini, P. Manfrinetti, A. Palenzona, D. Daghero, R. S. Gonnelli, and V. A. Stepanov, Phys. Rev. B 71, 144505 (2005).

${ }^{14}$ M. Angst, S. L. Bud'ko, R. H. T. Wilke, and P. C. Canfield, Phys. Rev. B 71, 144512 (2005).

${ }^{15}$ R. S. Gonnelli, D. Daghero, G. A. Ummarino, A. Calzolari, V. Dellarocca, V. A. Stepanov, S. M. Kazakov, J. Jun, and J. Karpinsky, cond-mat/0407267 (unpublished).

${ }^{16}$ A. A. Golubov, A. Brinkman, O. V. Dolgov, J. Kortus, and O. Jepsen, Phys. Rev. B 66, 054524 (2002); V. G. Kogan and N. V. Zhelezina, ibid. 69, 132506 (2004).

${ }^{17}$ P. F. Sullivan and G. Seidel, Phys. Rev. 173, 679 (1968).

${ }^{18}$ Note that in contrast to the $x=0$ and 0.1 samples the zero-field transition in transmittivity measurements is very large in the $x$ $\gtrsim 0.2$ samples and the complete screening is only obtained below $\sim 18 \mathrm{~K}$
${ }^{19}$ F. Bouquet, Y. Wang, I. Sheikin, T. Plackowski, A. Junod, S. Lee, and S. Tajima, Phys. Rev. Lett. 89, 257001 (2002).

${ }^{20}$ M. E. Zhitomirsky and V. H. Dao, Phys. Rev. B 69, 054508 (2004).

${ }^{21}$ V. G. Kogan and N. V. Zhelezina, Phys. Rev. B 71, 134505 (2005).

${ }^{22}$ E. Zeldov, A. I. Larkin, V. B. Geshkenbein, M. Konczykowski, D. Majer, B. Khaykovich, V. M. Vinokur, and H. Shtrikman, Phys. Rev. Lett. 73, 1428 (1994); E. H. Brandt, Phys. Rev. B 59, 3369 (1999).

${ }^{23}$ J. R. Clem, Supercond. Sci. Technol. 5, 33 (1992).

${ }^{24}$ E. H. Brandt, Phys. Rev. B 68, 054506 (2003).

${ }^{25}$ M. Zehetmayer, M. Eisterer, J. Jun, S. M. Kazakov, J. Karpinski, and H. W. Weber, Phys. Rev. B 70, 214516 (2004); M. Eisterer, M. Zehetmayer, H. W. Weber, and J. Karpinski, Phys. Rev. B 72, 134525 (2005).

${ }^{26}$ F. Bouquet, Y. Wang, R. A. Fisher, D. G. Hinks, J. D. Jorgensen, A. Junod, and N. E. Phillips, Europhys. Lett. 56, 856 (2001).

${ }^{27}$ A. Y. Liu, I. I. Mazin, and J. Kortus, Phys. Rev. Lett. 87, 087005 (2001).

${ }^{28}$ G. E. Blonder, M. Tinkham, and T. M. Klapwijk, Phys. Rev. B 25, 4515 (1982).

${ }^{29}$ J. Kortus, O. V. Dolgov, R. Kremer, and A. A. Golubov, Phys. Rev. Lett. 94, 027002 (2005). 